\title{
Annealed disorder, rare regions, and local moments: A novel mechanism for metal-insulator transitions
}

\author{
D. Belitz, ${ }^{1}$ T.R. Kirkpatrick, ${ }^{2}$ and Thomas Vojta ${ }^{1,3}$ \\ ${ }^{1}$ Department of Physics and Materials Science Institute, University of Oregon, Eugene, OR 97403 \\ ${ }^{2}$ Institute for Physical Science and Technology, and Department of Physics, University of Maryland, College Park, MD 20742 \\ ${ }^{3}$ Institut für Physik, TU Chemnitz, D-09107 Chemnitz, Germany
}

(July 3, 2018)

\begin{abstract}
It is shown that for noninteracting electron systems, annealed magnetic disorder leads to a new mechanism, and a new universality class, for a metal-insulator transition. The transition is driven by a vanishing of the thermodynamic density susceptibility rather than by localization effects. The critical behavior in $d=2+\epsilon$ dimensions is determined, and the underlying physics is discussed. It is further argued that annealed magnetic disorder, in addition to underlying quenched disorder, describes local magnetic moments in electronic systems.
\end{abstract}

PACS numbers: 71.30.+h; 64.60.Ak

Metal-insulator transitions (MITs) remain a fascinating and only incompletely understood phenomenon [i]. Conceptually, one distinguishes between Anderson transitions in models of noninteracting electrons, and MottHubbard transitions of clean, interacting electrons. At the former, the electronic charge diffusivity $D$ is driven to zero by quenched, or frozen-in, disorder, while the thermodynamic properties do not show critical behavior. At the latter, the thermodynamic density susceptibility $\partial n / \partial \mu$ vanishes due to electron-electron interaction effects. In either case, the conductivity $\sigma=(\partial n / \partial \mu) D$ vanishes at the MIT. In many real systems both quenched disorder and interactions are present, which makes a theoretical understanding of the resulting Anderson-Mott transition very difficult. One particular complication is provided by the presence of magnetic local moments (LMs) in such systems. There is much experimental evidence for LMs [2], and their formation has been studied theoretically [3], but no existing theory can describe their interplay with the transport properties near the MIT [1]. Another complication is the possible presence of annealed disorder, which is in thermal equilibrium with the rest of the system and hence involves disorder averaging of the partition function. This is in contrast to quenched disorder that requires an averaging of the free energy, which is usually done by means of the replica trick [4.

In this Letter we make two contributions to the MIT problem. (1) We show that annealed disorder leads to a MIT that belongs to none of the previously studied classes. It is driven by a vanishing $\partial n / \partial \mu$ and thus resembles a Mott-Hubbard transition, even if no correlation effects are explicitly considered. (2) We propose a mechanism by which additional annealed disorder is generically self-generated in quenched disordered systems, and we argue that a type of LMs can be described in terms of it. We further develop a method for incorporating these 'annealed LMs' into a transport theory.

Let us start by considering Wegner's nonlinear sigmamodel $(\mathrm{NL} \sigma \mathrm{M})$ [5] for noninteracting electrons with non- magnetic quenched disorder. The action reads

$$
\mathcal{A}=\frac{-1}{2 G} \int d \mathbf{x} \operatorname{tr}(\nabla Q(\mathbf{x}))^{2}+2 H \int d \mathbf{x} \operatorname{tr}(\Omega Q(\mathbf{x})) .
$$

Here $Q(\mathbf{x})$ is a matrix field that comprises two fermionic degrees of freedom. Accordingly, $Q$ carries two Matsubara frequency indices $n$ and $m$, and two replica indices $\alpha$ and $\beta$ to deal with the quenched disorder. The matrix elements $Q_{n m}^{\alpha \beta}$ are spin-quaternion valued to allow for particle-hole and spin degrees of freedom. It is convenient to expand them in a basis $\tau_{r} \otimes s_{i}(r, i=0,1,2,3)$ where $\tau_{0}=s_{0}$ is the $2 \times 2$ unit matrix, and $\tau_{1,2,3}=-s_{1,2,3}=$ $-i \sigma_{1,2,3}$, with $\sigma_{j}$ the Pauli matrices. For simplicity, we will ignore the particle-particle or Cooper channel, which amounts to dropping $\tau_{1}$ and $\tau_{2}$ from the spin-quaternion basis [1]. $Q$ is subject to the constraints $Q^{2}(\mathbf{x}) \equiv 1$, and $\operatorname{tr} Q(\mathbf{x}) \equiv 0 . \Omega_{n m}^{\alpha \beta}=\delta_{n m} \delta_{\alpha \beta} \Omega_{n}\left(\tau_{0} \otimes s_{0}\right)$ is a frequency matrix with $\Omega_{n}=2 \pi T n$ a bosonic Matsubara frequency and $T$ the temperature. $G$ is a measure of the disorder that is proportional to the bare resistivity, and the frequency coupling $H$ is proportional to the bare density of states at the Fermi level. tr denotes a trace over all discrete degrees of freedom that are not shown explicitly.

The properties of this model are well known [5, 6, 1]. The bare action describes diffusive electrons, with $D=$ $1 / G H$ the diffusion coefficient. Under renormalization $D$ decreases with increasing disorder until a MIT is reached at a critical disorder value. The critical behavior is known in an $\epsilon$-expansion about the lower critical dimension $d=$ 2. In the absence of the Cooper channel, the MIT appears only at two-loop order at a critical disorder strength of $O(\sqrt{\epsilon}) . H$, which determines the specific heat coefficient, the spin susceptibility, and $\partial n / \partial \mu$, is uncritical, which makes this MIT an Anderson transition.

Now we add magnetic annealed disorder to the model. Since our general results are independent of its origin, we first proceed without specifying it. Annealed disorder implies that the $Q$ in the resulting terms all carry the same replica index [4]; otherwise, the functional form of 
the resulting additional terms in the action can be taken from Ref. [6]. We obtain two additional terms, viz.

$$
\begin{aligned}
\Delta \mathcal{A}^{(1)}=\frac{T M_{1}}{8} \sum_{\alpha} \int & d \mathbf{x} \sum_{j=1}^{3}\left[\operatorname{tr}\left(\left(\tau_{3} \otimes s_{j}\right) Q^{\alpha \alpha}(\mathbf{x})\right)^{2}\right. \\
- & \left.\operatorname{tr}\left(Q^{\alpha \alpha}(\mathbf{x})\right)^{2}\right]
\end{aligned}
$$

and $\Delta \mathcal{A}^{(2)}=\Delta \mathcal{A}^{(2, s)}+\Delta \mathcal{A}^{(2, t)}$, where

$$
\begin{aligned}
\Delta \mathcal{A}^{(2, j)}= & \frac{T M_{2}^{j}}{8} \sum_{\alpha \neq \beta} \sum_{n m} \sum_{r=0,3} \int d \mathbf{x}\left[\operatorname{tr}\left(\tau_{r} \otimes s_{i}\right) Q_{n m}^{\alpha \beta}(\mathbf{x})\right] \\
& \times\left[\operatorname{tr}\left(\tau_{r}^{\dagger} \otimes s_{i}\right) Q_{m n}^{\beta \alpha}(\mathbf{x})\right],
\end{aligned}
$$

with $j=s$ for $i=0$ (spin-singlet), and $j=t$ for $i=1,2,3$ (spin-triplet). $\Delta \mathcal{A}^{(2)}$ arises from the need to absorb the scattering rate due to the annealed disorder in $G$. The coupling constants $M_{1}, M_{2}^{s}$, and $M_{2}^{t}$ are related to the strength of the magnetic annealed disorder. The factor of $T$ appears naturally in front of any annealed disorder term, a crucial point that we will come back to later.

The action $\mathcal{A}+\Delta \mathcal{A}^{(1)}+\Delta \mathcal{A}^{(2)}$ can be analyzed by standard means. Note that the mass terms in Eqs. (2) are proportional to temperature, making them quite different from conventional masses due to quenched disorder. In many respects, they are similar to electron-electron interaction terms in a Q-field theory formalism [7]. We denote the renormalized coupling constants that correspond to $G, H, M_{1}$, and $M_{2}^{s, t}$ by $g, h, m_{1}$, and $m_{2}^{s, t}$, and define $\delta_{1,2}^{s, t}=m_{1,2}^{s, t} / h$. The renormalization group (RG) flow equations to one-loop order are

$$
\begin{aligned}
& \frac{d g}{d l}=-\epsilon g+g^{2}\left(\delta_{2}^{s}+3 \delta_{2}^{t}-3 \delta_{1}\right) \\
& \frac{d h}{d l}=-h g\left(\delta_{2}^{s}+3 \delta_{2}^{t}-3 \delta_{1}\right) \\
& \frac{d \delta_{1}}{d l}=-g\left[-4 \delta_{1}^{2}+\delta_{1}\left(\delta_{2}^{s}+3 \delta_{2}^{t}\right)+\left(\delta_{2}^{s}-\delta_{2}^{t}\right)^{2}\right], \\
& \frac{d \delta_{2}^{s}}{d l}=g\left[3 \delta_{1}^{2}+3 \delta_{1}\left(\delta_{2}^{s}-2 \delta_{2}^{t}\right)-3 \delta_{2}^{t}\left(\delta_{2}^{s}-\delta_{2}^{t}\right)\right], \\
& \frac{d \delta_{2}^{t}}{d l}=g\left[3 \delta_{1}^{2}-\delta_{1}\left(2 \delta_{2}^{s}+\delta_{2}^{t}\right)-\left(\delta_{2}^{s}\right)^{2}\right. \\
&\left.-2\left(\delta_{2}^{t}\right)^{2}+3 \delta_{2}^{s} \delta_{2}^{t}\right]
\end{aligned}
$$

where $l=\ln b$ with $b$ the RG length scale factor. Besides unstable fixed points (FPs), there is a line of critical fixed points (FPs) $\left(g^{*}, h^{*}, \delta_{1}^{*}, \delta_{2}^{s *}, \delta_{2}^{t *}\right)=\left(\epsilon / 4 \delta_{2}^{*}, 0,0, \delta_{2}^{*}, \delta_{2}^{*}\right)$ that correspond to an MIT (all of these FPs belong to the same universality class). Linearization about any of these FPs yields one relevant eigenvalue $\lambda_{g}=\epsilon+O\left(\epsilon^{2}\right)$ that determines the correlation length exponent $\nu=$ $1 / \lambda_{g}$, one marginal eigenvalue that corresponds to moving along the line of FPs, and two irrelevant eigenvalues equal to $-\epsilon+O\left(\epsilon^{2}\right)$. The anomalous dimension of $h$ is $\kappa=-\epsilon+O\left(\epsilon^{2}\right)$. In addition, the critical behavior of the single-particle density of states (DOS), $N$, at the Fermi level can be obtained from the wavefunction renormalization. Choosing the critical exponent of the DOS, $\beta$, the correlation length exponent $\nu$, and the dynamical critical exponent $z=d+\kappa$ as independent exponents, we find

$$
\nu=1 / \epsilon+O(1), \beta=\epsilon+O\left(\epsilon^{2}\right), z=2+O\left(\epsilon^{2}\right) .
$$

For the conductivity exponent we find $s=\nu \epsilon=1+O(\epsilon)$, and $\partial n / \partial \mu$, the spin susceptibility $\chi_{s}$, and the specific heat coefficient $\gamma=C_{V} / T$, which we collectively denote by $\chi$, all vanish with a critical exponent determined by $\kappa$. The diffusion coefficient, on the other hand, has no anomalous dimension and thus is uncritical to one-loop order, as can be seen from Eqs. (3a, 3b). With $t$ the dimensionless distance from the critical point at $T=0$, and $E$ the energy, we can summarize the critical behavior of these quantities by the homogeneity laws

$$
\begin{aligned}
\chi(t, T) & =b^{\kappa} \chi\left(t b^{1 / \nu}, T b^{z}\right), \\
N(t, T, E) & =b^{-\beta / \nu} N\left(t b^{1 / \nu}, T b^{z}, E b^{z}\right), \\
\sigma(t, T) & =b^{-s / \nu} \sigma\left(t b^{1 / \nu}, T b^{z}\right), \\
D(t, T) & =b^{-(s / \nu+\kappa)} D\left(t b^{1 / \nu}, T b^{z}\right) .
\end{aligned}
$$

We conclude that the MIT is driven by the vanishing of $\partial n / \partial \mu$, and therefore is qualitatively different from the localization transition that is found in the absence of annealed disorder. Indeed, putting $M_{1}=M_{2}^{s}=M_{2}^{t}=0$ we find that all thermodynamic anomalies disappear, as does the one-loop correction to $g$. At two-loop order, one finds instead a MIT of Anderson type [1].

We now turn to a specific realization, via local magnetic moments, of the annealed disorder that leads to the striking effects discussed above. To explain the salient points, it is easiest to initially consider a simpler field theory than the $Q$-matrix theory studied above, and adapt a classical line of reasoning from Ref. [8] to quantum field theories. Accordingly, we consider a scalar quantum field $\phi(\mathbf{x}, \tau)$ and an action

$$
S[\phi]=\int d x\left(\phi \partial_{\tau} \phi-\mathcal{H}[\phi, \nabla \phi]\right),
$$

Here $x=(\mathbf{x}, \tau)$ comprises position $\mathbf{x}$ and imaginary time $\tau, \int d x \equiv \int d \mathbf{x} \int d \tau, \mathcal{H}$ is a Hamiltonian density, and we use units such that $\hbar=k_{\mathrm{B}}=1$. We will assume that $S$ describes a phase transition from a disordered to an ordered phase, and will use a magnetic language, referring to $\langle\phi\rangle$ as 'magnetization'. Suppose that $\mathcal{H}$ contains quenched disorder of random-mass type, and that we are in the nonmagnetic phase, $\langle\phi\rangle=0$. The key idea is to not integrate out the quenched disorder as a first step, as one does in a conventional treatment [4], but rather to work with a particular disorder realization. Due to the quenched disorder there will be regions in space that energetically favor local order, $\langle\phi\rangle \neq 0$, even though there is no global order. Deep inside the disordered phase these 
regions will be rare, but in an arbitrarily large system we will find arbitrarily large rare regions with a finite probability. The action $S$ will then have static saddlepoint solutions $\Phi(\mathbf{x})$ that have a nonvanishing value of the magnetization only in the rare regions. Let there be $N$ such rare regions and associated local blobs of magnetization or LMs. Then we can actually construct $2^{N}$ such saddle points, which differ only by the way the sign of the magnetization is distributed among the LMs. Since the LMs are far apart, the energy differences between these $2^{N}$ saddle points will be small. In expanding about the saddle points, we therefore have no reason to prefer one of them over any of the others. Furthermore, since the LMs are self-generated by the system, albeit in response to the quenched potential, we assume that they are in thermal equilibrium with all other degrees of freedom as well as with each other. To calculate the partition function $Z$ it is therefore necessary to take into account fluctuations in the vicinity of each of the $2^{N}$ saddle points [9]:

$$
Z \approx \sum_{a=1}^{2^{N}} \int_{<} D[\varphi] \exp \left(-S\left[\Phi^{(a)}+\varphi\right]\right)
$$

Here $\int_{<} D[\varphi]$ denotes an integration over small fluctuations $\varphi$ in the vicinity of each of the saddle points. Notice that this restriction to small fluctuations is necessary in order to avoid double counting. Conversely, if we could perform the integral over the fluctuations exactly, then it would be sufficient to expand about one of the saddle points. In practice, however, one is restricted to a perturbative evaluation of the functional integral, and Eq. (7) is a good approximation [11].

We now consider the thermodynamic limit. Then the discrete set of $2^{N}$ saddle points turns into a saddlepoint manifold $\mathcal{M}(\Phi)$ that needs to be integrated over. Splitting off the saddle-point part of the action, $S[\phi]=$ $S[\Phi]+\Delta S[\Phi, \varphi]$, we have

$$
Z=\int D[\Phi] P[\Phi] \int D[\varphi] \exp (-\Delta S[\Phi, \varphi])
$$

with the probability distribution $P$ given by

$$
P[\Phi]=\mathcal{S}(\Phi) \exp \left(-\frac{1}{T} \int d \mathbf{x} \mathcal{H}[\Phi, \nabla \Phi]\right) .
$$

Here $\mathcal{S}$ denotes the support of the saddle-point manifold $\mathcal{M}$. Notice the factor of $1 / T$ in the exponent, which results from the static nature of the saddle points $[12]$.

In general it is not possible to determine $P[\Phi]$ explicitly. However, if we perform the $\Phi$ integration by means of a cumulant expansion, the most relevant term in the effective action will be the one that results from the term quadratic in $\mathcal{H}[\Phi]$ and the linear coupling between $\Phi$ and $\varphi^{2}$ in $\Delta S$. To obtain the most relevant term in the effective theory for the fluctuations $\varphi$, we thus can write, with $w>0$ a number [12],

$$
\begin{aligned}
Z \approx & \int D[\varphi] e^{-S[\varphi]} \int D[\Phi] \exp \left(\frac{-1}{w T} \int d \mathbf{x} \Phi^{2}(\mathbf{x})\right) \\
& \times \exp \left(\int d x \Phi(\mathbf{x}) \varphi^{2}(x)\right)
\end{aligned}
$$

Equation (9) is the partition function one would obtain by expanding perturbatively about just one of the saddle points, with static, annealed disorder appearing in addition to the quenched disorder still contained in $S[\varphi]$. The annealed disorder is governed by a Gaussian distribution whose variance is proportional to $T$. This property reflects the fact that the annealed disorder, as classical degrees of freedom in equilibrium with the rest of the system, must come with a Boltzmann weight, and it is the reason for the factors of $T$ in Eqs. (2).

Let us now explain how these arguments can be applied to the $Q$-field theory of interacting electrons to arrive at the action, Eqs. (11, 2). The magnetization is proportional to the expectation value $\left\langle\operatorname{tr}\left(\tau_{3} \otimes s_{i}\right) Q(\mathbf{x})\right\rangle[\mathbf{7 ]}$, and in the presence of quenched disorder that favors the formation of magnetic LMs, the exact fermionic theory that underlies the NL $\sigma \mathrm{M}$ [7] allows for saddle-point solutions where these components of $Q$ are locally nonzero and play the role of the field $\Phi$ above. This is in addition to a globally nonzero $\left\langle\operatorname{tr}\left(\tau_{0} \otimes s_{0}\right) Q(\mathbf{x})\right\rangle$ which reflects a nonvanishing DOS. By following the above reasoning for a scalar field, and going through the derivation of the sigma-model again, one obtains Eqs. (1,2).

We conclude with several remarks. First, we emphasize that we have studied a simplified model, neglecting both the Cooper channel and the electron-electron interaction. The latter point requires some clarification. In order to generate the annealed disorder from LMs, some interaction is necessary, (1) for local magnetic order to develop, and (2) in order for our canonical averaging over the saddle points to make physical sense. A truly noninteracting system would not sample all of these field configurations. Put differently, interactions make the energy barriers between the saddle points, which are infinite in a noninteracting system, finite and thus allow for an equilibration of the saddle-point degrees of freedom [9,11]. We have simplified our model by assuming points (1) and (2) above to be the only effect of the interactions. Of course, if the annealed disorder were due to some other mechanism, then our results would also apply to strictly noninteracting electrons. Clearly, one can study generalizations of our model. In addition to adding an explicit interaction term, one can restore the Cooper channel, which will make the FP we found compete with the ordinary localization FP that also occurs at one-loop order. In systems with time reversal symmetry, one then expects the MIT studied here to get preempted by a localization transition if the bare dimensionless mass $M_{2} / H$ is smaller than a number of $O(1)$. It would also be interesting to consider the present model to 2-loop order to see whether the diffusion coefficient will still not be renormalized (apart from 
the 'diffuson' localization contributions that will appear at that order), and whether the line of FPs gives way to a more conventional FP structure. These questions will be considered in the future.

Second, we point out that the strong effects of annealed disorder we found are characteristic of quantum statistical mechanics. In a classical scalar field theory, the leading term in the action generated upon integrating out annealed disorder is of the form (see Eq. (9)) $-\int d \mathbf{x} \varphi^{4}(\mathbf{x})$. It thus has the same form as the ordinary $\varphi^{4}$-term and is in general not very interesting (although it can lead, e.g., to a first order phase transition). In a quantum system, on the other hand, integrating out the annealed disorder yields $-\int d \mathbf{x} \int d \tau d \tau^{\prime} \varphi^{2}(\mathbf{x}, \tau) \varphi^{2}\left(\mathbf{x}, \tau^{\prime}\right)$, which has a different time structure than the usual $\varphi^{4}$ term. It is the extra time integral that makes the annealed disorder term more relevant than in the classical case.

Third, we come back to the fact that the variance of the Gaussian distribution for the annealed disorder is linear in $T$. If one used a Gaussian distribution with a temperature independent width, one would encounter factors of $1 / T$ in perturbation theory that force one to scale the annealed disorder strength with $T$ to obtain a meaningful theory. Annealed disorder with an unbounded distribution and a finite variance at $T=0$ is unphysical, since it allows the system to lower its energy arbitrarily far by digging itself a deeper and deeper trough. The necessity of the factor of $T$ was realized in Ref. [10], but its origin was not recognized 13.

Finally, let us explain why annealed disorder leads to a critical $\partial n / \partial \mu$, while quenched disorder without electron-electron interactions does not. To see this, we realize that annealed disorder essentially means potential troughs that are somewhat flexible, i.e. they adjust in response to the electrons. Let the sytem be in equilibrium at some value of the chemical potential $\mu$, and change $\mu$ slightly. Then the flexible potential will adjust, and as a result fewer electrons will have to flow out of or into the grand canonical reservoir than would be the case in the absence of annealed disorder. This explains why there is a correction to $\partial n / \partial \mu$ in perturbation theory. Furthermore, the diffusive dynamics of the electrons lead to this correction being a frequency-momentum integral over diffusion propagators, which is logarithmically singular in 2- $d$. In $d=2+\epsilon$ this leads to a critical $\partial n / \partial \mu$, as it happens with other quantities that are singular in perturbation theory in 2- $d$. This is the only known mechanism for a critical $\partial n / \partial \mu$ at a MIT in low-dimensional systems [14]. The recent observation of a critical $\partial n / \partial \mu$ at a $2-d$ MIT $\mid 15]$ is therefore very interesting in this context, even though our current theory does not describe a MIT in $d=2$.

This work was initiated at the Aspen Center for Physics, and supported by the NSF under grant Nos. DMR-98-70597 and DMR-96-32978, and by the DFG under grant No. SFB 393/C2.
[1] For a review, see, e.g., D. Belitz and T.R. Kirkpatrick, Rev. Mod. Phys. 66, 261 (1994).

[2] M.A. Paalanen, S. Sachdev, R.N. Bhatt, and A.E. Ruckenstein, Phys. Rev. Lett. 57, 2061 (1986); M.A. Paalanen, J.E. Graebner, R.N. Bhatt, and S. Sachdev, Phys. Rev. Lett. 61, 597 (1988); Y. Ootuka and N. Matsunaga, J. Phys. Soc. Japan 59, 1801 (1990).

[3] M. Milovanovic, S. Sachdev, and R.N. Bhatt, Phys. Rev. Lett. 63, 82 (1989), and references therein.

[4] See, e.g., G. Grinstein, in Fundamental Problems in Statistical Mechanics VI, E.G.D. Cohen (ed.), North Holland (Amsterdam, 1985).

[5] F. Wegner, Z. Phys. B 35, 207 (1979). We use the fermionic formulation of the model given in Ref. [6]. See also Ref. [河.

[6] K.B. Efetov, A.I. Larkin, and D.E. Khmelnitskii, Zh. Eksp. Teor. Fiz. 79, 1120 (1980) [Sov. Phys. JETP 52, 568 (1980)].

[7] D. Belitz and T.R. Kirkpatrick, Phys. Rev. B 56, 6513 (1997); D. Belitz, T.R. Kirkpatrick, and F. Evers, Phys. Rev. B 58, 9710 (1998).

[8] Viktor Dotsenko, A.B. Harris, D. Sherrington, and R.B. Stinchcombe, J. Phys. A 28, 3093 (1995).

[9] Elsewhere we have argued that for noninteracting LMs, the typical barriers between different saddle point states diverge in the bulk limit 10]. We expect that one effect of interactions between the LMs is to make the barriers finite, so that the averaging discussed here applies.

[10] R. Narayanan, T. Vojta, D. Belitz, and T.R. Kirkpatrick, Phys, Rev. B 60, 10150 (1999).

[11] The basic assumption underlying these arguments is that typical pairs of saddle-point configurations are separated by finite barriers [9], but not perturbatively accessible from one another. This amounts to an assumption about a separation of time scales that we expect to be true in some, but not all, systems.

[12] If one uses a simple saddle-point theory of noninteracting LMs for $\Phi$ in the Hamiltonian density in Eq. (8b), then the coefficient of $1 / T$ is found to be positive, indicating that the LM state is a thermodynamic equilibrium state. We expect a more realistic treatment, including LM interactions, to weaken the effects of these LMs and make the state thermodynamically unstable, changing the sign of the $1 / T$ term to the one used in Eqs. (8b,9), which physically makes these LMs dynamic rare fluctuations.

[13] Developing the theory without the explicit factor of $T$ in Eqs. (2) leads to Eqs. (3) with an extra factor of $1 / T$ on the righ-hand side. The only physical fixed point then requires the masses to scale with $T$. The factor of $T$ in Eqs. (2) is thus unavoidable on technical grounds, even if its presence on physical grounds is not realized.

[14] In high dimensions an order parameter theory of the Anderson-Mott transition yields a critical $\partial n / \partial \mu$, T.R. Kirkpatrick and D. Belitz, Phys. Rev. Lett. 73, 862 (1994), while in $d=2+\epsilon, \partial n / \partial \mu$ is uncritical [1].

[15] S.C. Dultz and H.W. Jiang, cond-mat/9909314. 